\title{
Cataract Surgery in Germany: Data from a German Registry on Quality Outcomes for Cataract and Refractive Surgery in the Year 2018
}

\author{
Christian Schäferhoff ${ }^{*}{ }^{*}$, Jörg Förster ${ }^{2}$, Bernd Schneider ${ }^{3}$ \\ ${ }^{1}$ Augenpraxisklinik Vogelsang, Esslingen am Neckar, Germany \\ ${ }^{2}$ Förster Projektlinien, Kropp, Germany \\ ${ }^{3}$ Numeras GmbH, Frankfurt am Main, Germany \\ Email: *christian@schaeferhoff.biz
}

How to cite this paper: Schäferhoff, C., Förster, J. and Schneider, B. (2020) Cataract Surgery in Germany: Data from a German Registry on Quality Outcomes for Cataract and Refractive Surgery in the Year 2018. Open Journal of Ophthalmology, 10, 297-306.

https://doi.org//10.4236/ojoph.2020.104033

Received: August 24, 2020

Accepted: November 7, 2020

Published: November 10, 2020

Copyright $\odot 2020$ by author(s) and Scientific Research Publishing Inc. This work is licensed under the Creative Commons Attribution International License (CC BY 4.0).

http://creativecommons.org/licenses/by/4.0/

(c) (i) Open Access

\begin{abstract}
Background: The German registry for cataract surgery was established in 2014. The main aim of this registry was to improve cataract surgery outcomes. Aim: The aim of this project is to offer a tool for benchmarking through the establishment of a reference database in Germany where surgeons could record and analyze their own outcomes. Methods: Data were collected between January 2018 and December 2018. The analyzed data included parameters of surgical technique, implanted intraocular lens (IOL), refractive and visual outcomes. Results: During the year of 2018, the German registry included 10,035 lens exchange surgeries and the complete follow-up was available for 9882 lens extractions. Approximately one third of the patients were between 76 and 80 years old and $60.6 \%$ of the cases had a best-corrected distance visual acuity (CDVA) before surgery of 0.5 or better. Parabulbar anesthesia was reported in $60.4 \%$ of the cases and phacoemulsification with implantation of a posterior chamber IOL was the chosen method in $92.2 \%$ of the cases. The power of the implanted IOL was between $21.0-22.9 \mathrm{D}$ in $29.4 \%$ of eyes. Surgical complications were reported in only 413 cases. After surgery, a CDVA of 0.5 or better was achieved in $90.8 \%$ of the cases. Most of the operated patients (64\%) had a residual refractive error within $\pm 0.5 \mathrm{D}$ ( $95 \%$ confidence interval 63.2 - 65.1). Conclusions: Our results show that the registry was implemented successfully with results comparable to the ones reported in EUREQUO.
\end{abstract}

\section{Keywords}

German Registry, Cataract Surgery, Visual Outcome, EUREQUO 


\section{Introduction}

A registry is an organized system for the collection, storage, retrieval and analysis of data for individual persons with a particular condition [1]. Through the use of registries, it is possible to increase the value of healthcare and enable medical professionals to identify and share best clinical practices, which could lead to lower costs [2].

A German registry for Cataract Surgery was implemented in 2014, and in 2018, more than 10,000 cataract and refractive lens exchange surgeries were reported in Germany. The exchange of the opacified natural lens by an artificial intraocular lens (IOL) is one of the most frequently performed surgeries worldwide [3]. In the recent past years, the number of available IOL models increased drastically, not only widening the options for patient care, but also the difficulties in the selection of the appropriate IOL for each patient [3].

The German registry was designed according to the proposed outcomes framework for cataract surgery which enables international comparison, helping to improve outcomes [4]. This registry was implemented with the following purposes: benchmarking for surgeons, benchmarking for IOL manufacturers, information of IOL design parameters on visual outcome, information of surgical parameters on visual outcome, fulfillment of medical device resolution requirements with regards to post marketing clinical follow-up, and reference for quality control and benchmarking in Germany.

The main goal of this project was to improve the treatment and standards of care for cataract and refractive surgery in Germany and to offer a tool for benchmarking through the establishment of a reference database in Germany where surgeons could record and analyze their own outcomes. To our best knowledge, this is the first registry on cataract surgery based solely on German data.

The purpose of this paper is to report the results of the German Cataract surgery database from the year 2018.

\section{Materials and Methods}

\subsection{Patients and Assessments}

The Annual Report on Cataract Surgery Germany (ARCSG) started in 2014. Ophthalmic surgeons across Germany were invited to contribute data on their cataract surgeries. Data collection includes data in quality control and benchmark tools on preoperative, intraoperative and postoperative parameters. In all the surgeries, the type of IOL was documented and consequently parameters as optic design, size, edge design, haptic design and material are available.

The data presented here have been derived from the German registry for Cataract Surgery. Data have been entered into the system from January 2, 2018, and were included in the analysis, reporting data in 2018.

\subsection{Statistical Analysis}

Results are reported as percentages with $95 \%$ Confidence Interval (95\%CI) or as 
mean \pm standard deviation (SD).

\section{Results}

The German registry included a total of 10,035 lens exchange surgeries (cataract and clear lens exchange) in 2018. Complete follow-up data were available for 9,882 lens extractions and the mean time from date of surgery to data of follow-up was 30 days.

\subsection{Preoperative Parameters}

Of the total included patients, $57.9 \%$ were females. Regarding age groups, more than one third of the patients belonged to the age group between $76-80$ years old, followed by the age group $71-75$ years old with approximately $23 \%$ of operated patients in 2018 (Figure 1).

The preoperative corrected distance visual acuity (CDVA) was $0.5(10 / 20)$ or better in $60.6 \%$ of the cases. CDVA was $0.1(6 / 60)$ or worse in $6.4 \%$ of all the cases. The values of preoperative axial length varied between $19.09 \mathrm{~mm}$ and $29.99 \mathrm{~mm}$ with a mean \pm SD of $23.41 \pm 1.19 \mathrm{~mm}$. The range of anterior chamber depth was $1.56-5.63 \mathrm{~mm}$ with a mean $\pm \mathrm{SD}$ of $3.08 \pm 0.42 \mathrm{~mm}$.

Approximately $64 \%$ of the patients undergoing lens exchange surgeries had some ocular comorbidities: $9.7 \%$ had glaucoma, $10.9 \%$ maculopathy, $1.0 \%$ diabetic retinopathy, $0.9 \%$ amblyopia and $41.7 \%$ had another ocular disease.

\subsection{Intraoperative Parameters}

The main type of anesthesia reported was peribulbar anesthesia in $60.4 \%$ of the cases. Retrobulbar anesthesia was used in $22.4 \%$ of the cases and drip anesthesia was applied in $15.6 \%$ of the surgeries.

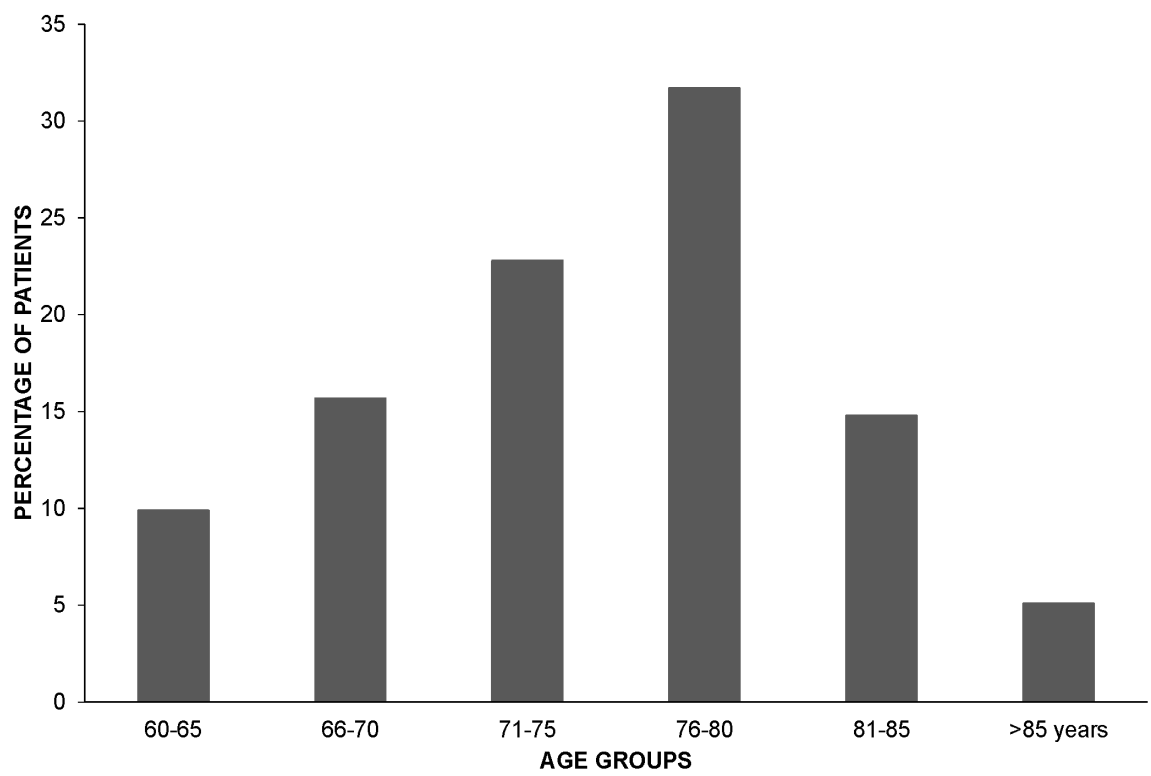

Figure 1. Percentage of the operated patients in the different age groups. 
In the majority of cases, $96.2 \%$, the chosen method of surgery was phacoemulsification with implantation of a posterior chamber IOL. The other reported surgical methods were femtosecond laser-assisted cataract extraction (3.6\%), planned extracapsular cataract extraction (0.03\%) and other type of surgeries $(0.12 \%)$. A sulcus implantation was performed in $0.33 \%$ of the cases and an anterior chamber IOL was implanted in $0.05 \%$ of the surgeries.

The type of incision performed during surgery for lens exchange varied. In most of the cases, $76.4 \%$, clear cornea incisions were reported, followed by corneoscleral (18.2\%), limbal (5.3\%), being clear cornea/limbal incisions the less frequently reported type of incision $(0.1 \%)$.

Percentages of implanted IOL power are shown in Figure 2. In $29.4 \%$ of the eyes, the power of implanted IOL was between 21.0 - 22.9 D. Aspheric IOLs were implanted in $67.8 \%$ of the eyes and 411 (5\%) natural lenses were replaced by IOLs with additional features: toric IOLs in 247 eyes, multifocal IOLs (diffractive, multifocal and trifocal) in 141 eyes and toric multifocal IOLs in 23 eyes.

Surgeons reported intraoperative difficulties in $12.6 \%$ of the surgeries: narrow pupil in $7.5 \%$, shallow anterior chamber in $3.3 \%$, mature cataract in $1.4 \%$, previous retinal surgery in $0.3 \%$ and previous vitrectomy in $0.1 \%$. Beyond these difficulties, surgical complications have also been reported. These complications were only reported in 413 cases and included posterior capsule rupture with or without vitreous loss, dropped nucleus, damage, prolapse or trauma of the iris.

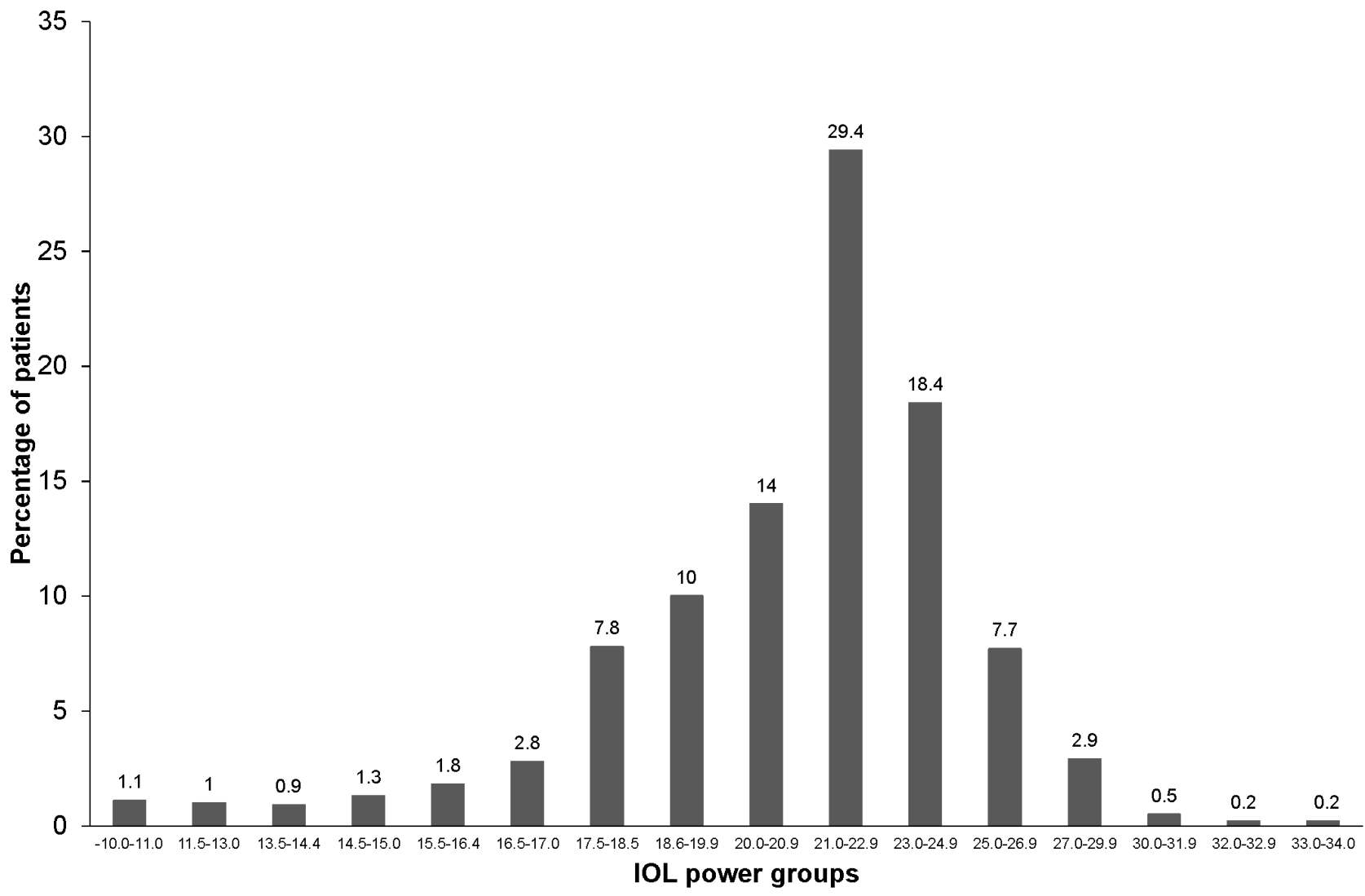

Figure 2. Power of the implanted IOLs shown as percentages. 


\subsection{Postoperative Data (Outcome at Follow-Up Visits)}

Figure 3 shows the percentages of eyes reaching different levels of CDVA (decimal) preoperatively and at the last postoperative visit. A CDVA of $1.0(6 / 6)$ or better at the last follow-up visit was achieved by $42.9 \%$ (95\%CI $42.0-43.8)$ of all reported cases. A CDVA of $0.5(6 / 12)$ or better was achieved by $90.8 \%(95 \% \mathrm{CI}$ 90.2 - 91.3) of all cases.

The evolution of sphere, cylinder and residual refractive error (SEQ) preoperatively and at each postoperative visit is shown in Figure 4. The mean \pm SD of SEQ was $-0.13 \pm 0.83 \mathrm{D}$ and the absolute mean \pm SD of SEQ was $0.52 \pm 0.66 \mathrm{D}$.

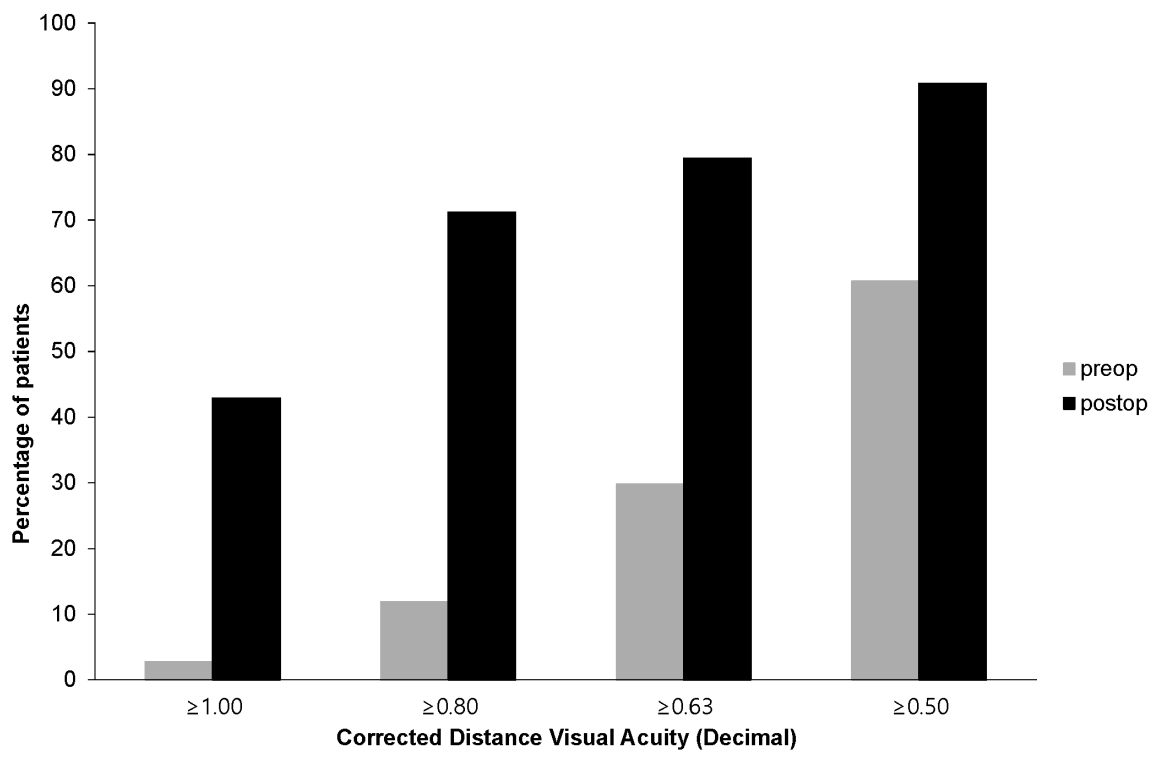

Figure 3. Percentage of eyes reaching different levels of CDVA (decimal). Preop-preoperative; postop-last postoperative visit.

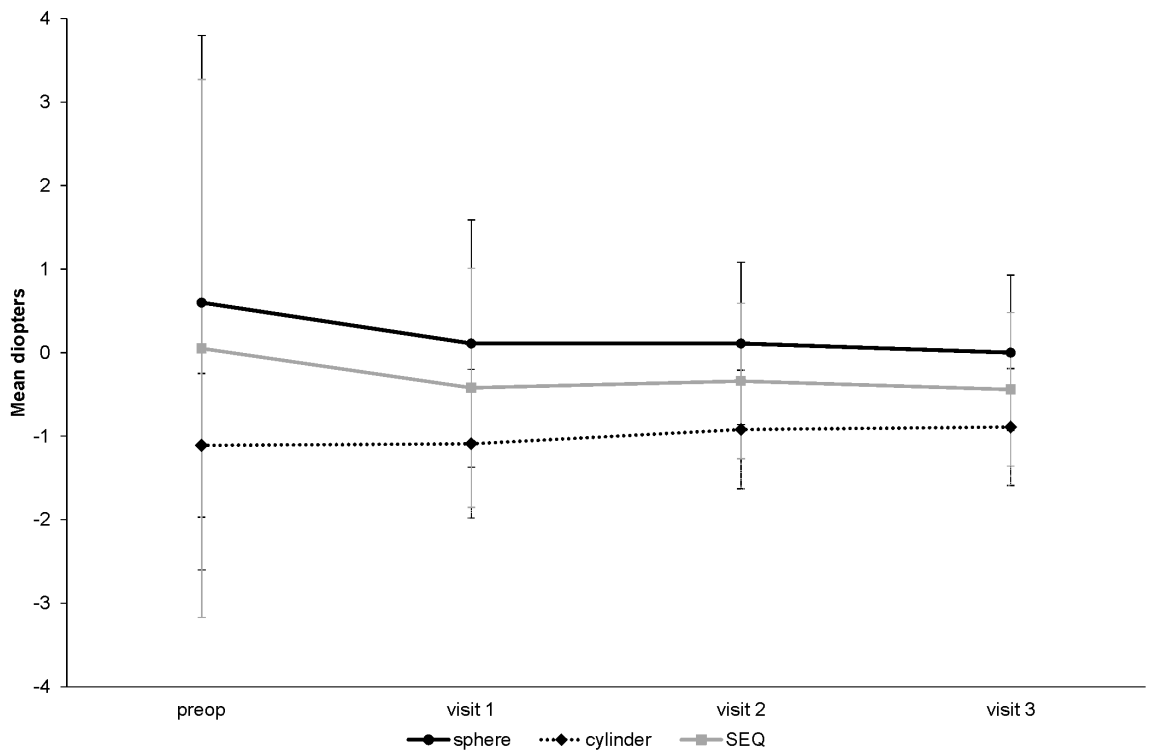

Figure 4. Evolution of sphere, cylinder and residual refractive error at preoperative and at each postoperative visit. 
When comparing the targeted and achieved spherical equivalent, approximately $64 \%$ of the evaluated eyes had a residual refractive error within $\pm 0.50 \mathrm{D}$ (95\%CI 63.2 - 65.1) and 89.9\% were within $\pm 1.0 \mathrm{D}$ (95\%CI 89.3 - 90.5). Approximately $10 \%$ of the evaluated eyes had a residual refractive error greater than 1.00D (Figure 5).

The reported postoperative complication rate was very low during the follow-up period: in 25 cases there was a persistent corneal edema and an uncontrolled elevated intraocular pressure was reported in 22 cases. Endophthalmitis occurred in three cases.

\section{Discussion}

The number of registries in ophthalmology has increased over the last years. A recently published review paper [1] identified 97 clinical eye registries and 8 of them included data regarding cataract extraction or refractive surgery; three of these registries are data from pediatric cataract [4]-[11]. Apart from the German registry presented in this paper, there are three more national databases originated in India (Aravind Eye Hospital cataract registry) [12], Malaysia (Malaysian National Cataract registry) [11] and Sweden (Swedish National Cataract Register) [8]. There are also two multinational registries: EUREQUO, comprising 18 European countries and Australia [5] [13]. The National Eyecare Outcomes Network (NEON), which originated in the USA, is a multinational registry that includes 32 states from the USA, Australia, France, Trinidad and Tobago [7]. From these databases, the Swedish register was the first to be implemented in 1992, followed by the USA register that started in 1996. Visual acuity is a parameter evaluated by all the registries [1].

Comparing the mean age of patients undergoing cataract surgery, the available data show that 70 years is the approximately reported mean age, with the

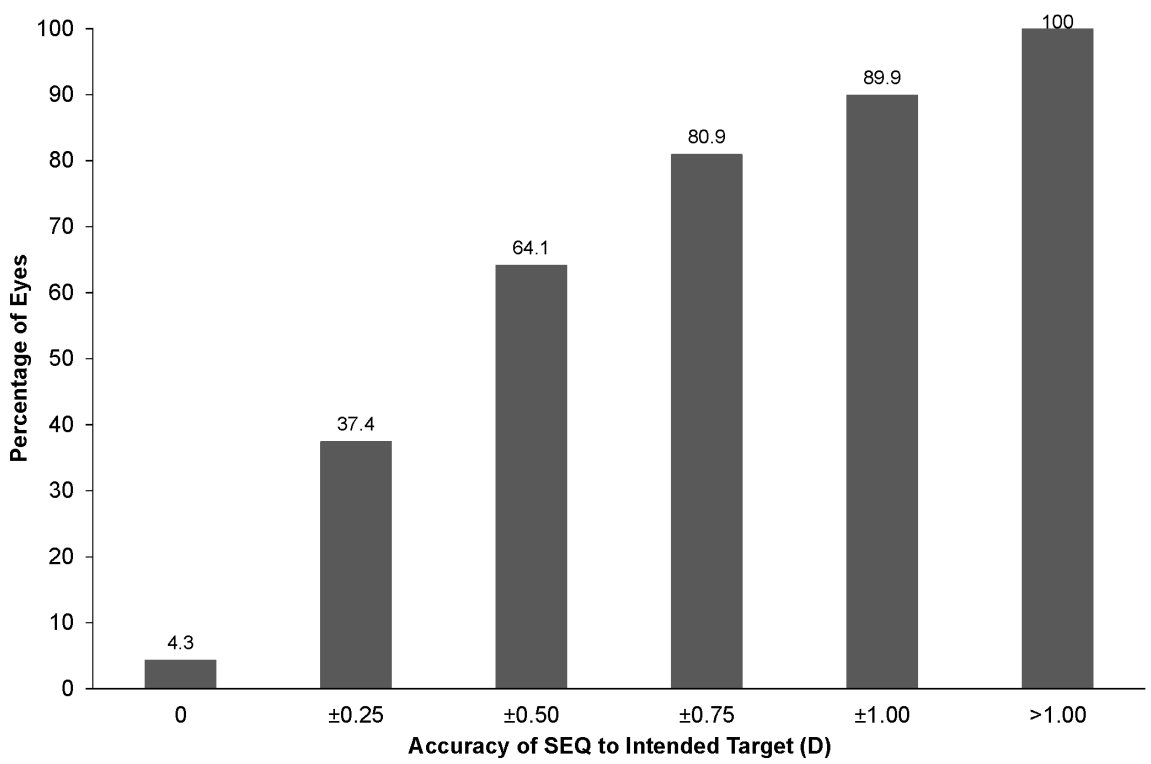

Figure 5. Percentage of patients with different levels of residual refractive error. 
exception of the Malaysian database that reported a mean age of 64 years [5] [7] [8] [11] [12] [14]. Another consistent fact is the percentage of women, approximately $60 \%$ [7] [11], which could be related to age: due to the longer life expectancy of women, the dominance of females is expectable [13].

There are different types of anesthesia used for cataract extraction. In the report of the two years' experience of the National Eyecare Outcomes Network Cataract Surgery Database in 2000, retrobulbar anesthesia was the most frequently used type of anesthesia [7] whilst in the Indian database, all the surgeries were performed under topic or retrobulbar anesthesia [12]. In the Swedish database, the type of anesthesia was not included in the report since almost all surgeries were performed under topical anesthesia. In the EUREQUO database, topical anesthesia was the most frequent (37.9\%), followed by sub-tenon $(26.2 \%)$ and retrobulbar (18.3\%) [13]. The frequencies of used anesthetic techniques vary among the different databases, which is in accordance with what was reported in EUREQUO: a significant variation in the anesthetic techniques among the different clinics [13].

In our study, phacoemulsification was the surgical technique performed in almost all of the cases (96.2\%), which is very similar to the reports from other databases: $99.5 \%$ in EUREQUO [13] and 92.3\% in NEON [7]. On the contrary, in India, the rate of phacoemulsification is much lower, only $26 \%$ [12].

Although femtosecond laser-assisted cataract surgery was only used in $3.6 \%$ of the surgeries in our report, it was the second most frequently used technique. Femtosecond laser-assisted cataract surgery was used in cataract surgery for the first time in 2008, with the expectation that it would yield superior outcomes compared to phacoemulsification surgery. Both techniques, phacoemulsification and femtosecond laser-assisted cataract surgery, appear to be safe and effective and there are a significant number of surgeons worldwide that do not consider the latter approach to be more beneficial or cost-effective [15] [16]. However, more recent data from EUREQUO showed that, in 3379 cases of femtosecond laser-assisted cataract surgeries, visual and refractive outcomes of patients treated with this technique were more favorable compared to manual phacoemulsification [17].

A crucial issue in cataract surgery is the accurate calculation of the IOL power. The expectations of patients to be spectacle-free have been increasing steadily over the last years, with patients being more demanding regarding their outcomes after surgery. Although different mathematical formulas have been developed for IOL power calculation, resulting in more precise refractive outcomes [18], this issue is far from being solved. Cataract surgeons constantly evaluate the outcomes after surgery for a more personalized lens calculation formula to achieve better visual and refractive outcomes [3] [19].

Our data revealed a complete follow-up for 9882 lens extractions. Visual and refractive outcomes are the ones that will allow comparing our results to other reported results as a measure of quality control. In fact, an alignment of outcome 
measures for cataract surgery is crucial for comparison across contexts [4].

With a mean follow-up of 30 days, our results are comparable to those described in other published databases. Concerning CDVA, $90.8 \%$ of our patients achieved a post-operative CDVA of 0.5 (decimal). Eighty-nine percent of the NEON patients achieved a best corrected visual acuity of 0.5 (decimal) [7]. In the Indian database, approximately $90 \%$ of the patients that underwent cataract surgery achieved a 1 month post-operative CDVA of at least 0.5 (decimal) [12]. In the EUREQUO database, $94.4 \%$ of operated eyes achieved a CDVA of 0.5 (decimal) or better between 7 - 60 days after surgery. The same database reported $97.2 \%$ in cases of no ocular comorbidities [13] [20].

Regarding refractive outcomes, in $49 \%$ of the NEON patients, the difference between the target and the achieved spherical equivalent was within $0.5 \mathrm{D}$ and $29 \%$ was between 0.5 and 1.0D [7]. The EUREQUO database reported a mean prediction error of $0.55 \mathrm{D}$ [13], which is very similar to our results. Our results also show that $64.1 \%$ of the patients have a refractive error within $\pm 0.5 \mathrm{D}$ and $89.9 \%$ of the patients have a refractive error within $\pm 1.0 \mathrm{D}$, which is considered to be a good outcome [18]. Thus, the outcomes reported in our German registry can be considered as good and realistic, according to what is expected from patients with these characteristics and subject to lens exchange surgery.

\section{Limitations}

This type of registry is not mandatory in Germany and any standardization of this type of study does not exist. Therefore, our data might not represent the "real-world" in Germany. Other limitations are the unstructured selection of documenting doctors, and the short mean follow-up time, reporting only 1-year data.

\section{Conclusion}

Our preoperative, intraoperative, visual and refractive outcomes from surgeries in Germany are comparable to the ones reported in other registries, namely EUREQUO, which is the registry with the most recent data and with a closer geographic location to ours.

\section{Conflicts of Interest}

The authors declare no conflicts of interest regarding the publication of this paper.

\section{References}

[1] Tan, J.C.K., Ferdi, A.C., Gillies, M.C. and Watson, S.L. (2019) Clinical Registries in Ophthalmology. Ophthalmology, 126, 655-662. https://doi.org/10.1016/j.ophtha.2018.12.030

[2] Larsson, S., Lawyer, P., Garellick, G., Lindahl, B. and Lundström, M. (2012) Use of 13 Disease Registries in 5 Countries Demonstrates The Potential to Use Outcome Data to Improve Health Care's Value. Health Affairs, 31, 220-227. 
https://doi.org/10.1377/hlthaff.2011.0762

[3] Davis, G. (2016) The Evolution of Cataract Surgery. Missouri Medicine, 113, 58-62.

[4] Mahmud, I., Kelley, T., Stowell, C., Haripriya, A., Boman, A., Kossler, I., Morlet, N., Pershing, S., Pesudovs, K., Goh, P.P., Sparrow, J.M. and Lundström, M. (2015) A Proposed Minimum Standard Set of Outcome Measures for Cataract Surgery. JAMA Ophthalmology, 133, 1247-1252. https://doi.org/10.1001/jamaophthalmol.2015.2810

[5] Lundström, M., Manning, S., Barry, P., Stenevi, U., Henry, Y. and Rosen, P. (2015) The European Registry of Quality Outcomes for Cataract and Refractive Surgery (EUREQUO): A Database Study of Trends in Volumes, Surgical Techniques and Outcomes of Refractive Surgery. Eye and Vision, 2, Article No. 8. https://doi.org/10.1186/s40662-015-0019-1

[6] Ho, S.F., Adnan, T.H.B. and Goh, P.P. (2017) Prevalence and Factors Associated with Second Eye Cataract Surgery and the Trend in the Time Interval between the Two Eye Surgeries Based on the Malaysian National Eye Database. Asia-Pacific Journal of Ophthalmology (Philadelphia, PA), 6, 310-317.

[7] Lum, F., Schein, O., Schachat, A.P., Abbott, R.L., Hoskins Jr., H.D. and Steinberg, E.P. (2000) Initial Two Years of Experience with the AAO National Eyecare Outcomes Network (NEON) Cataract Surgery Database. Ophthalmology, 107, 691-697. https://doi.org/10.1016/S0161-6420(99)00184-0

[8] Lundström, M., Stenevi, U. and Thorburn, W. (2002) The Swedish National Cataract Register: A 9-Year Review. Acta Ophthalmologica Scandinavica, 80, 248-257. https://doi.org/10.1034/j.1600-0420.2002.800304.x

[9] Repka, M.X., Dean, T.W., Lazar, E.L., Yen, K.G., Lenhart, P.D., Freedman, S.F., Hug, D., Rahmani, B., Wang, S.X., Kraker, R.T., Wallace, D.K. and Pediatric Eye Disease Investigator Group (2016) Cataract Surgery in Children from Birth to Less than 13 Years of Age: Baseline Characteristics of the Cohort. Ophthalmology, 123, 2462-2473. https://doi.org/10.1016/j.ophtha.2016.09.003

[10] Haargaard, B., Nyström, A., Rosensvärd, A., Tornqvist, K. and Magnusson, G. (2015) The Pediatric Cataract Register (PECARE): Analysis of Age at Detection of Congenital Cataract. Acta Ophthalmologica, 93, 24-26.

https://doi.org/10.1111/aos.12445

[11] Salowi, M., Goh, P.P., Lee, M.Y., Adnan, T. and Ismail, M. (2015) The Malaysian Cataract Surgery Registry: Profile of Patients Presenting for Cataract Surgery. Asia-Pacific Journal of Ophthalmology (Philadelphia, PA), 4, 191-196. https://doi.org/10.1097/APO.0000000000000068

[12] Haripriya, A., Chang, D.F., Reena, M. and Shekhar, M. (2012) Complication Rates of Phacoemulsification and Manual Small-Incision Cataract Surgery at Aravind Eye Hospital. Journal of Cataract \& Refractive Surgery, 38, 1360-1369.

[13] Lundström, M., Barry, P., Henry, Y., Rosen, P. and Stenevi, U. (2012) Evidence-Based Guidelines for Cataract Surgery: Guidelines Based on Data in the European Registry of Quality Outcomes for Cataract and Refractive Surgery Database. Journal of Cataract \& Refractive Surgery, 38, 1086-1093.

[14] Lundström, M., Goh, P.P., Henry, Y., Salowi, M.A., Barry, P., Manning, S., Rosen, P. and Stenevi, U. (2015) The Changing Pattern of Cataract Surgery Indications: A 5-Year Study of 2 Cataract Surgery Databases. Ophthalmology, 122, 31-38. https://doi.org/10.1016/j.ophtha.2014.07.047

[15] Abell, R.G., Kerr, N.M. and Vote, B.J. (2013) Femtosecond Laser-Assisted Cataract Surgery Compared with Conventional Cataract Surgery. Clinical \& Experimental 
Ophthalmology, 41, 455-462. https://doi.org/10.1111/ceo.12025

[16] Abell, R.G. and Vote, B.J. (2014) Cost-Effectiveness of Femtosecond Laser-Assisted Cataract Surgery versus Phacoemulsification Cataract Surgery. Ophthalmology, 121, 10-16. https://doi.org/10.1016/j.ophtha.2013.07.056

[17] Lundström, M., Dickman, M., Henry, Y., Manning, S., Rosen, P., Tassignon, M.J., Young, D., Stenevi, U. and European Society of Cataract and Refractive Surgeons Femtosecond Laser-Assisted Cataract Surgery Study Collaborators (2017) Femtosecond Laser-Assisted Cataract Surgeries Reported to the European Registry of Quality Outcomes for Cataract and Refractive Surgery: Baseline Characteristics, Surgical Procedure, and Outcomes. Journal of Cataract and Refractive Surgery, 43, 1549-1556.

[18] Aristodemou, P., Knox Cartwright, N.E., Sparrow, J.M. and Johnston, R.L. (2011) Formula Choice: Hoffer Q, Holladay 1, or SRK/T and Refractive Outcomes in 8108 Eyes after Cataract Surgery with Biometry by Partial Coherence Interferometry. Journal of Cataract \& Refractive Surgery, 37, 63-71.

[19] Hoffer, K.J. and Savini, G. (2017) IOL Power Calculation in Short and Long Eyes. Asia-Pacific Journal of Ophthalmology (Philadelphia, PA), 6, 330-331.

[20] Manning, S. and Barry, P. (2014) Benefits of the European Registry of Quality Outcomes for Cataract and Refractive Surgery to an Ophthalmology Trainee: How an Ophthalmology Trainee Used EUREQUO to Audit Their Surgical Training. Journal of Cataract \& Refractive Surgery, 40, 157-159. 